University of Nebraska - Lincoln

DigitalCommons@University of Nebraska - Lincoln

U.S. Environmental Protection Agency Papers

U.S. Environmental Protection Agency

2007

\title{
Sound Survey Designs can Facilitate Integrating Stream Monitoring Data Across Multiple Programs
}

\author{
David P. Larsen \\ U.S. Environmental Protection Agency, larsen.phil@epa.gov \\ Anthony R. Olsen \\ U.S. Environmental Protection Agency \\ Steven H. Lanigan \\ USDA Forest Service \\ Chris Moyer \\ USDI Bureau of Land Management \\ Kim K. Jones \\ Oregon Department of Fish and Wildlife \\ See next page for additional authors
}

Follow this and additional works at: https://digitalcommons.unl.edu/usepapapers

Larsen, David P.; Olsen, Anthony R.; Lanigan, Steven H.; Moyer, Chris; Jones, Kim K.; and Kincaid, Thomas M., "Sound Survey Designs can Facilitate Integrating Stream Monitoring Data Across Multiple Programs" (2007). U.S. Environmental Protection Agency Papers. 143.

https://digitalcommons.unl.edu/usepapapers/143

This Article is brought to you for free and open access by the U.S. Environmental Protection Agency at DigitalCommons@University of Nebraska - Lincoln. It has been accepted for inclusion in U.S. Environmental Protection Agency Papers by an authorized administrator of DigitalCommons@University of Nebraska - Lincoln. 


\section{Authors}

David P. Larsen, Anthony R. Olsen, Steven H. Lanigan, Chris Moyer, Kim K. Jones, and Thomas M. Kincaid 


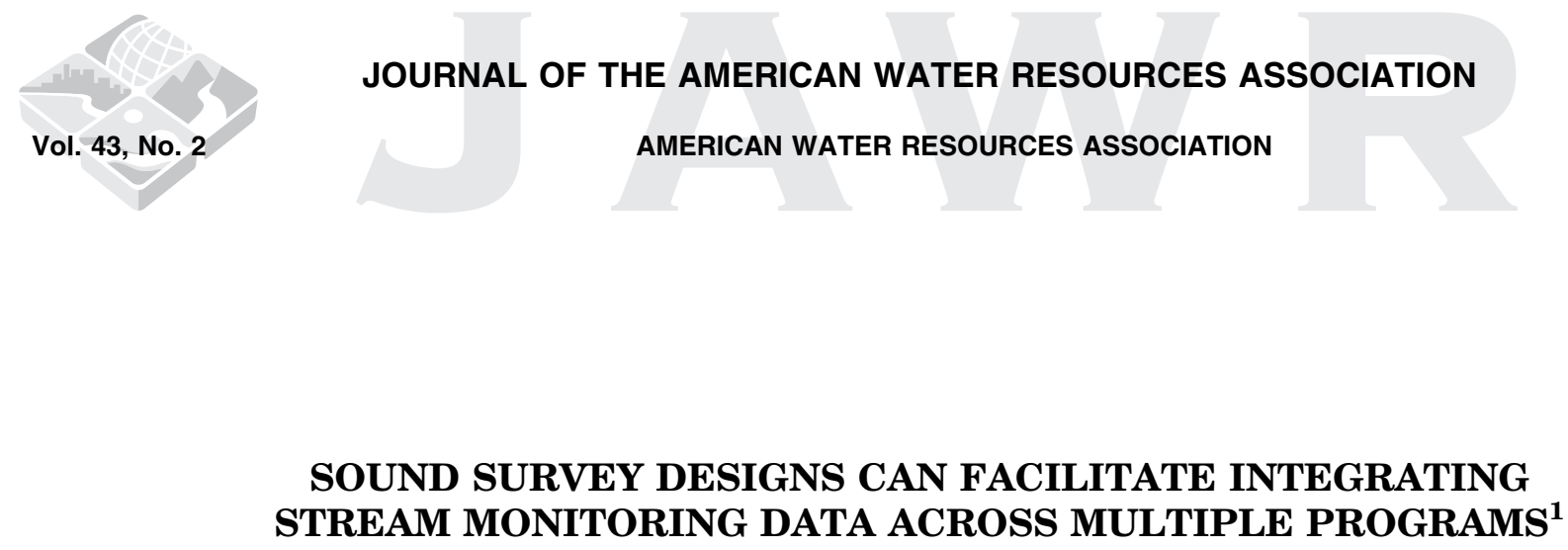

David P. Larsen, Anthony R. Olsen, Steven H. Lanigan, Chris Moyer, Kim K. Jones, and Thomas M. Kincaid ${ }^{2}$

\begin{abstract}
Multiple agencies in the Pacific Northwest monitor the condition of stream networks or their watersheds. Some agencies use a stream "network" perspective to report on the fraction or length of the network that either meets or violates particular criteria. Other agencies use a "watershed" perspective to report on the health or condition of watersheds. The agencies often use the same indicators and measurement protocols for data collection and often conduct monitoring in overlapping geographic regions. In these situations, agencies would like to combine data across different monitoring studies in a statistically sound manner to make regional estimates of condition. Three statistical survey design principles will facilitate combining such studies: (1) a clearly specified statistical target population of interest, including elements that comprise the population, (2) a consistent representation of that target population (such as a digital map of the stream network and watersheds), and (3) rules that incorporate randomization to guide the selection of the sample of sites on which measurements will be made. A case study illustrates the application of these design principles using two agency monitoring programs interested in combining stream channel data for different purposes: one for making network summaries and the other for evaluating watershed condition.
\end{abstract}

(KEY TERMS: monitoring; rivers/streams; statistics; stream surveys; stream condition; watershed condition.)

Larsen, David P., Anthony R. Olsen, Steven H. Lanigan, Chris Moyer, Kim K. Jones, and Thomas M. Kincaid, 2007. Sound Survey Designs Can Facilitate Integrating Stream Monitoring Data Across Multiple Programs. Journal of the American Water Resources Association (JAWRA) 43(2):384-397. DOI: 10.1111/j.1752-1688.2007. 00030.x

\section{INTRODUCTION}

Agreement in principle often conceals differences in details. Thorough discussions on a topic reveal those differences, which can be substantial. So it is with monitoring the condition of riverine and riparian ecosystems. There is often substantial general agreement about the need to monitor the condition of these systems, whether under the guise of ecosystem health,

\footnotetext{
${ }^{1}$ Paper No. J05005 of the Journal of the American Water Resources Association (JAWRA). Received January 20, 2005; accepted May 10, 2006. (C) 2007 American Water Resources Association. No claim to original U.S. government works.

${ }^{2}$ Respectively, Aquatic Biologist, Pacific States Marine Fisheries Commission, c/o Western Ecology Division, National Health and Ecological Effects Research Lab, Office of Research and Development, U.S. EPA, 200 SW 35th St., Corvallis, Oregon 97333; Environmental Statistician, Western Ecology Division, National Health and Ecological Effects Research Lab, Office of Research and Development, U.S. EPA, 200 SW 35th St., Corvallis, Oregon 97333; Fisheries Biologist, Aquatic and Riparian Effectiveness Monitoring Program, USDA Forest Service, Pacific NW Region, Resource Planning; Fisheries Biologist, Aquatic and Riparian Effectiveness Monitoring Program, Monitoring, 333 SW First Ave., Portland, Oregon 97204; Aquatic Ecologist, USDI Bureau of Land Management, 4077 Research Way, Corvallis, Oregon 97333; Conservation and Recovery Program, Oregon Department of Fish \& Wildlife, 28655 Hwy 34, Corvallis, Oregon 97333; and Statistician, Western Ecology Division, National Health and Ecological Effects Research Lab, Office of Research and Development, U.S. EPA, 200 SW 35th St., Corvallis, Oregon 97333 (E-Mail/Larsen: larsen.phil@epa.gov).
} 
aquatic health, riverine and riparian integrity, or watershed health/integrity. However, these interests are often couched in specific terms that suggest some differences in perspectives. For example, agencies like Oregon's Department of Environmental Quality and Washington's Department of Ecology are required under the Clean Water Act to report on the condition of aquatic resources based on the number of river kilometers that meet certain criteria, or that are impaired with respect to designated uses. Others focus instead on the condition of watersheds (Montgomery et al., 1995; Ator et al., 2003; Reeves et al., 2004; Detenbeck et al., 2005). However, underlying these differences is a fundamental common interest in protecting, preserving, and restoring the health or integrity of aquatic ecosystems.

Despite different perspectives, substantial agreement exists on the attributes to be used in evaluating riverine and riparian systems, including aspects of riverine and riparian biological condition, water quality, geomorphology, physical habitat structure, hydrology, and upslope characteristics. Often substantial agreement occurs on the specific indicators and measurement protocols to be used in the field. Making watershed assessments relies, in part, on the same stream channel measurements used to evaluate the condition of the stream network. Differences often occur in the interpretation of the measures of these aspects of riverine and riparian condition. How should the array of indicators be interpreted to make an assessment of watershed condition? How should the array of indicators be interpreted to evaluate attainment of designated uses? If data collected by one agency could be used easily by another agency for making regional estimates of condition, then the agencies could share data even though their ultimate interpretation, use, and spatial application of the data might differ. The potential for integration of stream monitoring data is based on this common interest in site scale measurements. Given that common protocols are used for the indicators of common interest, the adoption of three key survey design principles by agencies will enable monitoring data to be shared for these indicators to make inferences about regional condition.

Many agency objectives require evaluating the condition of riverine and riparian resources at broad spatial scales across the geographic domain under the management responsibility of particular agencies, e.g., across states, ecoregions, forests, or basins. A census of the resource at these scales is usually prohibitively expensive. If regional scale estimates are needed, and a census cannot be conducted on the resource, using sample surveys is an approach with a well founded theory in many dimensions of human interest (Kish, 1965; Converse, 1987; Thompson, 1992; Olsen et al.,
1999). The theory and practical dimensions of sample surveys as applied to aquatic resources provide a useful framework for facilitating the integration of monitoring data and information. Adopting some of the key principles of sample surveys for aquatic resource monitoring can go a long way toward encouraging coordinated monitoring, both improving the precision of estimates and saving money.

The objective here is to illustrate how adopting sample survey design principles can serve as a framework for the integration of data at regional scales on common indicators that are evaluated with the same protocols. The illustrative case study considers aquatic resources from two angles: one is the need to summarize information on the basis of the length of the riverine and riparian network (i.e., what proportion of the network achieves a particular criterion? or how many $\mathrm{km}$ of the stream network fail to meet designated uses?); the other is the need to summarize information on the basis of watershed condition (i.e., how many or what proportion of watersheds are in good condition?). To do this, some key sample survey principles are introduced and adapted for riverine and riparian systems. The case study applies these principles to summarize information on a riverine and riparian network basis (stream length) and on a watershed basis. The case study illustrates how sample survey designs can be developed in such a way that indicator data can be shared and then shows specifically how this is done with an illustrative indicator. Finally, the implications for the development of coordinated designs, and some of the pitfalls, are discussed.

Full integration of data across monitoring programs also relies on addressing other issues such as comparability of data including common specification of the attributes of interest, comparable measurement protocols, adequate training, and data quality. Many of these issues have been discussed elsewhere (e.g., Johnson et al., 2001). The purpose here is to focus on the design issues that if recognized and incorporated into aquatic monitoring programs allow statistically sound data integration, given data comparability.

\section{KEY FEATURES OF SAMPLE SURVEYS}

The theory of survey sampling is founded on the basic premise that a census on a specified target population of interest cannot be conducted cost effectively. Instead, a representative sample of the resource is drawn; measurements are made on the sample; then, the results are compiled and interpreted consistent with the specifics of the survey design to make relevant inferences about the target 
population. With respect to aquatic resources, this implies that a sample of the riverine and riparian network or a sample of watersheds of the specified size (or size range) is drawn using survey sampling procedures adapted for this type of natural resource. Measurements are then made at the sites in this sample of the resource, and inferences are drawn to the target population of the riverine and riparian network or to the population of watersheds from which the sample was drawn. Some examples of the application of survey designs to monitoring riverine and riparian systems include the following. Herlihy et al. (2000) drew a sample of sites in first to third Strahler order streams in the mid-Atlantic highlands region of the eastern U.S. Stevens (2002) describes a multiyear survey of Oregon's coastal streams that allows integration of the monitoring needs of two agencies with water resource responsibilities: Oregon's Department of Fish and Wildlife and Department of Environmental Quality. Ator et al. (2003) and Detenbeck et al. (2005) drew a sample of watersheds in the midAtlantic coastal plain and in West Virginia, respectively.

Other examples can be found at http://www.epa. gov/nheerl/arm, a website that describes many aspects of survey sampling for aquatic resources. In all of these cases, a regional target population of the resource of interest was defined and a digital coverage of points, stream networks, or polygons was used to represent the target population. Then a sample of a specified size was drawn from the coverage using survey sampling procedures. The designs for these surveys used an adaptation of the theory of survey sampling to address the peculiar aspects of aquatic resources (Stevens, 1997; Stevens and Olsen, 1999, 2004). Basic texts that develop the statistical foundation for survey designs and techniques for making inferences based on the designs, from which the following is drawn, include: Cochran (1987), Lohr (1999) and Thompson (1992).

The design and implementation of sample surveys require several decisions that ultimately guide specific designs. These include the following.

\section{A Specific Description of the Target Population to be Characterized and the Population Elements that Comprise the Target Population}

For riverine and riparian systems, a description of the target population includes specifying the geographic domain of interest. Is it statewide or multistate? Does it cover one or more ecoregions or river basins? It also requires specifying the riverine and riparian "population" and the population elements. For example, the elements could consist of (1) all watersheds of a specific size range in the geographic domain, (2) all locations in the network of Strahler first to third order perennial streams in the geographic domain, (3) all locations in the network of low gradient streams, (4) all locations in the network of unconstrained channels in the geographic domain, or (5) all locations in the network of perennial streams, regardless of size, in the geographic domain. For stream networks, each point on a stream may be considered as a population element, consistent with the view that stream networks are continuous resources. Or, the stream network can be decomposed into discrete segments, e.g., at confluences and/or at geomorphic breaks. Each segment would be considered a population element. Both are valid conceptual definitions for elements of the stream network target population (Cressie, 1991). For watersheds, each watershed of the specified type or size is a population element. The population elements should be described in such a way that field crews can recognize that a site selected in the sample is part of the target population.

\section{A Frame that Represents the Target Population}

A conceptual description of a target population requires translation into a specific form from which a sample can be drawn. The frame is this device from which a sample is drawn. The frame can be a list of the population elements or it can be a mapped representation of the target population. Usually for aquatic resources, a digital coverage, such as a digital representation of the stream network, serves as the frame, whether the target population is the stream network or a set of watersheds. A frame contains the collection of all possible population elements that are eligible for selection. In most cases, the population elements in the frame are the same as the elements in the target population. The frame should contain a faithful representation of the specified target population.

\section{Rules Incorporating Randomization that Direct the Selection of the Sample from the Frame}

A requirement of survey sampling is that each member of the target population (each population element) has a known positive probability of being included in the sample. It is not necessary for each element to have the same probability of selection, just that the selection probabilities are known. A simple example illustrates the process. Suppose that the population elements consist of all possible locations on a stream network of $1000 \mathrm{~km}$ and that the frame 
consists of a digital coverage of the stream network. Suppose Agency A chooses a simple random sample of 50 sites; each site then represents $1000 \mathrm{~km} / 50=20 \mathrm{~km}$; that is, has a weight of $20 \mathrm{~km}$ and an inclusion density (i.e., probability) of 0.05 . Suppose Agency B uses the same target population definition and the same frame and chooses a simple random sample of 20 sites. Then each site represents $1000 \mathrm{~km} / 20=50 \mathrm{~km}$ of stream length. The use of sample weights (inverse of the selection probabilities) allows for expansion of the sample's characteristics to describe the target population. The calculation of weights (or inclusion probabilities) for a study can be more complex but follows the general principle. For example, if an agency used a stratified random sample on the network, where one stratum consisted of $200 \mathrm{~km}$ and another stratum consisted of $800 \mathrm{~km}$ of stream length, then if 25 sites were selected within each stratum, the weights would be $200 \mathrm{~km} / 25=8 \mathrm{~km}$ and $800 \mathrm{~km} / 25=32 \mathrm{~km}$. Note that the sum of the weights for the sample equals $1000 \mathrm{~km}$, i.e., the length of the stream network.

\section{COMBINING SURVEYS}

Combining two or more surveys when the objective is to estimate regional condition involves two major aspects. First, the surveys must include one or more indicators in common that are comparable. If this is not the case, no basis exists to combine the surveys. Having indicators that are comparable implies either that the same field protocols, lab protocols, and indicator construction procedures are the same or that a comparability study has concluded that the indicators are measuring the same aspect of stream condition. Second, the surveys must be able to be re-interpreted as if they were conducted as a single probability survey design. Only the second aspect is considered further in this paper.

To combine two surveys requires (1) a combined target population definition, (2) a combined sample frame, and (3) a re-calculation of weights (inclusion densities or probabilities) using original survey design information. A combined target population definition is created from the two surveys' target population definitions. The key is to state the combined definition in terms of mutually-exclusive, exhaustive subsets of the stream resource. For example, suppose one survey defines their target population as all perennial streams within region $\mathrm{A}$ and the other as all perennial-coded first to fourth Strahler order (wadeable) streams within region $B$, which partially overlaps region $\mathrm{A}$. The combined target population is all per- ennial streams within region A plus all wadeable streams within the portion of region $B$ that is outside of region A. In some cases it may be of interest to restrict the combined target population to a subset of the original surveys' target population. An example might be all wadeable streams within the combined region defined by regions $\mathrm{A}$ and $\mathrm{B}$.

A combined sample frame is constructed from the two surveys' sample frames. An important feature is to add an attribute that assigns each location on the stream network to mutually-exclusive, exhaustive categories based on the combined target population definition and the original survey designs. For the example, assume that simple random samples were used in both regions. The categories for the combined sample frame attribute would be (1) all perennialcoded Strahler order stream segments in region A excluding the portion in region $\mathrm{B},(2)$ all wadeable stream segments in region $\mathrm{B}$ excluding the portion in region $\mathrm{A}$, (3) all wadeable stream segments that are in both regions, (4) all perennial-coded fifth and higher Strahler order (boatable) stream segments that are in both regions. Note that the latter two categories must be distinguished since the two survey designs did not sample the same Strahler order streams within the overlapping region. If stratification were used in the studies, then the categories must also keep the strata separate.

Re-calculation of weights for the combined study depends on the original survey designs and information from the combined sample frame. The combined inclusion probability for a unit, $u$, in the combined sample frame, $S$, can be determined as follows. Let $S_{\mathrm{A}}$ be a sample from sample frame $U_{\mathrm{A}}$ and $S_{\mathrm{B}}$ be a sample from sample frame $U_{\mathrm{B}}$, then $S \cup S_{\mathrm{A}} S_{\mathrm{B}}$ is a probability sample from $U \cup U_{\mathrm{A}} U_{\mathrm{B}}$ and the inclusion density for an element $u$ in $S$ is computed as

$$
\begin{aligned}
\pi_{u}= & P(u \in S)=P\left(u \in S_{\mathrm{A}}\right)+P\left(u \in S_{\mathrm{B}}\right) \\
& -P\left(u \in S_{\mathrm{A}}\right) \times P\left(u \in S_{\mathrm{B}}\right)
\end{aligned}
$$

and the weight for $u$ is $1 / \pi_{\mathrm{u}}$. Overton et al. (1993) give an example of calculating inclusion probabilities. Continuing the example above, assume 100, 100, 50, and $650 \mathrm{~km}$ of stream length is in region B only, wadeable both regions, boatable both regions, and region A only, respectively. Also that sample sizes of 25 in region $\mathrm{B}$ and 50 in region $\mathrm{A}$ were selected as a simple random sample. Then the inclusion densities for a stream location in region $\mathrm{B}$ only is $25 /(100+100)=0.125$, in wadeable both is $25 / 200+$ $50 /(100+50+650)-25 / 200 * 50 /(100+50+650)=$ 0.17969 , in boatable both is $50 /(100+50+650)=$ 0.0625 , and in region A only is $50 /(100+$ $50+650)=0.0625$. These give weights of $8,5.565$, 
16 , and $16 \mathrm{~km}$, respectively. An alternate approach for re-calculating weights that depends on the concept of post-stratification can be used but it requires knowing the sample sizes that actually occur in each of the unique categories. The computation continues to use Equation (1) applied to the unique categories and sample sizes occurring in each category.

Combining two probability surveys can be more complex. Two surveys may include different, but overlapping, geographic regions, or domains. They may use different criteria for stratification or unequal probability of selection. One survey may use a twostage survey design to select stream elements, while the other may not. Combining the surveys remains possible as long as the frames for both surveys are available and the details of each survey are known, i.e., how randomization was used in the selection of sample units.

\section{A CASE STUDY}

The following case study illustrates how survey design principles are used for combining data. The Oregon Department of Fish and Wildlife (ODFW) is interested in the condition of a stream network and the United States Department of Agriculture (USDA) Forest Service's Aquatic and Riparian Effectiveness Monitoring Program (AREMP; Reeves et al., 2004) is interested in the condition of small watersheds; the agencies geographic domains partially overlap. In both cases, a component of the monitoring program measures an array of stream channel physical habitat attributes. A relevant question is whether ODFW could make use of the measurements that AREMP takes in the ODFW domain, and whether AREMP could use measurements that ODFW takes in its domain, in each case for making population estimates over the agency's domain of interest. The result of combining data would be to make more precise estimates of the target resource than could be made based on each agencies respective data alone. Although both surveys were designed independently at different times to support long term monitoring (decades), they were designed with the above described principles in mind so that data could be combined in a statistically sound manner should the respective agencies wish to do so. Both designs relied on the same 1:100,000 scale digital stream network coverage as the frame material and both were designed following an adaptation of survey design principles to aquatic resources (Stevens, 1997; Stevens and Olsen, 1999, 2004). The specifics of each design are described next.

\section{The ODFW Design}

As part of the Oregon Plan for Salmon and Watersheds, an integrated monitoring program was designed to estimate abundances of adult coho spawners and juveniles, habitat and water quality, and the condition of resident biotic assemblages in Oregon's coastal streams (Nicholas, 1997; Oregon Plan, 1997; Stevens, 2002). The design covers five distinct monitoring areas along the coast of Oregon. The habitat portion of the surveys identified an array of channel and riparian characteristics to be measured or estimated (Jones and Moore, 1999; Moore et al., 1997). Approximately 50 sites are sampled for habitat attributes each year in each monitoring area for a sample size of about 250 sites per year. The target population consists of all possible locations (population elements) on the network of Strahler first to third order perennial streams in each of the five monitoring areas (with a few local exceptions). Digital versions of the USGS 1:100,000 scale hydrographic maps were used as the frame material. Each element of the stream network represented in the frame was selected with known probability.

\section{The AREMP Design}

The USDA Forest Service's Northwest Forest Plan (NFP) included a mandate to develop and implement monitoring programs addressing various topics of special interest e.g., spotted owl, marbled murrelet, late-successional and old-growth forests, watershed condition, and changes over time. For each of these topics, monitoring programs were developed. For watershed condition, AREMP advisors selected small watersheds with $>25 \%$ federal ownership as the appropriate scale of interest, covering the region of the NFP (parts of California, western Oregon, and western Washington; Reeves et al., 2004). These were operationally defined by refining the United States Geological Services (USGS) hydrologic accounting system (Legleiter, 2001; see also http://www.ncgc. nrcs.usca.gov/products/datasets/watershed) from its "level 4" to a "level 6" which AREMP chose for evaluating watershed condition. The target population consists of the approximately 1,400 sixth field hydrologic accounting units (sixth field HUs) that meet this definitional requirement in the NFP region. Each sixth field HU is a population element of interest to AREMP. Size ranges from about 1300 to 5000 ha.

For the pilot studies, a two-stage survey design was implemented. First, a sample of HUs was selected, and then a sample of locations on streams within these HUs was selected for those measurements made on streams. A sample of $250 \mathrm{HUs}$ was selected to be 
monitored over a period of 5 years, 50 each year, to be repeated in subsequent 5-year cycles for change and trend detection (Reeves et al., 2004). On each HU, indicators of upslope, riparian, and channel condition (habitat), and selected biological assemblages were to be measured or estimated. A decision support model was outlined as a way to aggregate the various indicators into an overall assessment of watershed condition (Reeves et al., 2004). Channel condition indicators included many of the same attributes that ODFW routinely surveys as part of its monitoring program. At the second stage within each HU, Strahler first to third order sites were selected randomly using the same basic procedure used to select the ODFW sites. The same frame material used for the ODFW site selection was also used for the AREMP site selection. On average, 5 to 6 sites within each HU were surveyed. This two-stage design allows the calculation of the probability of selection of any particular stream site within the target population of all possible stream locations within the $1,400 \mathrm{HUs}$.

\section{Linking $O D F W$ and AREMP Designs}

The survey design for ODFW results in a spatiallydistributed set of stream sites (the sample) across the stream network in each of the five monitoring areas. The survey design for AREMP produces a clustered sample of stream sites driven by the initial selection of HUs, then stream sites within HUs. Both designs rely on the selection of first to third order stream sites from the same frame material $(1: 100,000$ scale digital stream map), using probability methods for selection of sites with the elements being any point on the stream network. Combining the surveys requires recalculating inclusion probabilities and resultant weights for the areas where sampling overlaps (overlapping domains) to incorporate the increased sample sizes for these areas. Use of the same frame material, population elements, and probability sampling methods allows determination of the new weights as described next.

This illustrative case study draws from the ODFW coastal survey and the AREMP 2001 pilot survey. Because data on only 16 HUs comprising the AREMP pilot survey over the entire NFP region (in CA, OR, and WA) were available, only four of which fell within the ODFW domain, the data from AREMP's pilot survey were treated as if all 16 of the HUs were selected within the portion of the ODFW domain that AREMP overlaps. This was accomplished by randomly assigning the $12 \mathrm{HUs}$ to similar HUs within the ODFW domain. This step was done strictly for illustration purposes and will not be necessary when the monitoring programs have run for several years. Figure 1 illus- trates the ODFW domain and the part of the NFP domain that overlaps with the ODFW domain. The goal, strictly for this illustration, is to combine the stream data collected from the 16 AREMP pilot surveys (as if they all fell within the ODFW domain) with the ODFW survey results in two general ways: (1) combine the AREMP survey with the ODFW survey to characterize the coastal stream networks and (2) combine the ODFW survey with the AREMP survey to characterize the condition of HUs. In the first case, the elements of the target population are all locations in the stream network. In the second, the elements of the target population are the collection of HUs. The net effect, in both cases, is to increase the sample size that each agency could use in the areas where monitoring domains overlap. The process for combining data in both cases is described next.

When interest is in making statements about the characteristics of HUs, the probability of selecting HUs from both surveys must be determined. In the example below, HU condition is expressed as the average of the stream indicator scores measured at sites within the HU. Combining ODFW HU and AREMP $\mathrm{HU}$ data to describe $\mathrm{HU}$ condition requires recalculating $\mathrm{HU}$ inclusion probabilities and their respective weights. AREMP HUs are selected at the first stage, with known probability. However, ODFW directly selected stream sites without first selecting HUs. A key question then is: what is the probability of selecting a HU within which ODFW sites fall? This is calculated by associating ODFW sites with a sixth field HU to determine the HUs for which probability of selection must be determined and then calculating the stream length within each of the sixth field HUs. ODFW stratified by monitoring area, so the probability of selecting a HU within a monitoring area is obtained by dividing the HU stream length by the total stream length in a monitoring area. The weights are the reciprocal of the selection probability, normalized to the total number of HUs within the monitoring area. The ODFW design can now be reinterpreted as a HU-based survey in which the probability of selecting HUs is unequal. ODFW and AREMP surveys only overlap for HUs that are greater than $25 \%$ federal land. When a HU has less than $25 \%$ federal land, the probability of selection is the same as the probability that ODFW selected the HU. For HUs with greater than $25 \%$ federal land, the probability of selection, applying equation 1 , is:

$$
\begin{aligned}
& \text { Prob }(\mathrm{HU}) \\
& =\text { Prob }(\mathrm{HU} \text { in ODFW })+\text { Prob }(\mathrm{HU} \text { in AREMP }) \\
& \quad-\text { Prob }(\mathrm{HU} \text { in } \mathrm{ODFW}) \times \text { Prob }(\mathrm{HU} \text { in AREMP })
\end{aligned}
$$

When interest is in making statements about the characteristics of the stream network, the probability 
Larsen, Olsen, Lanigan, Moyer, Jones, and Kincaid

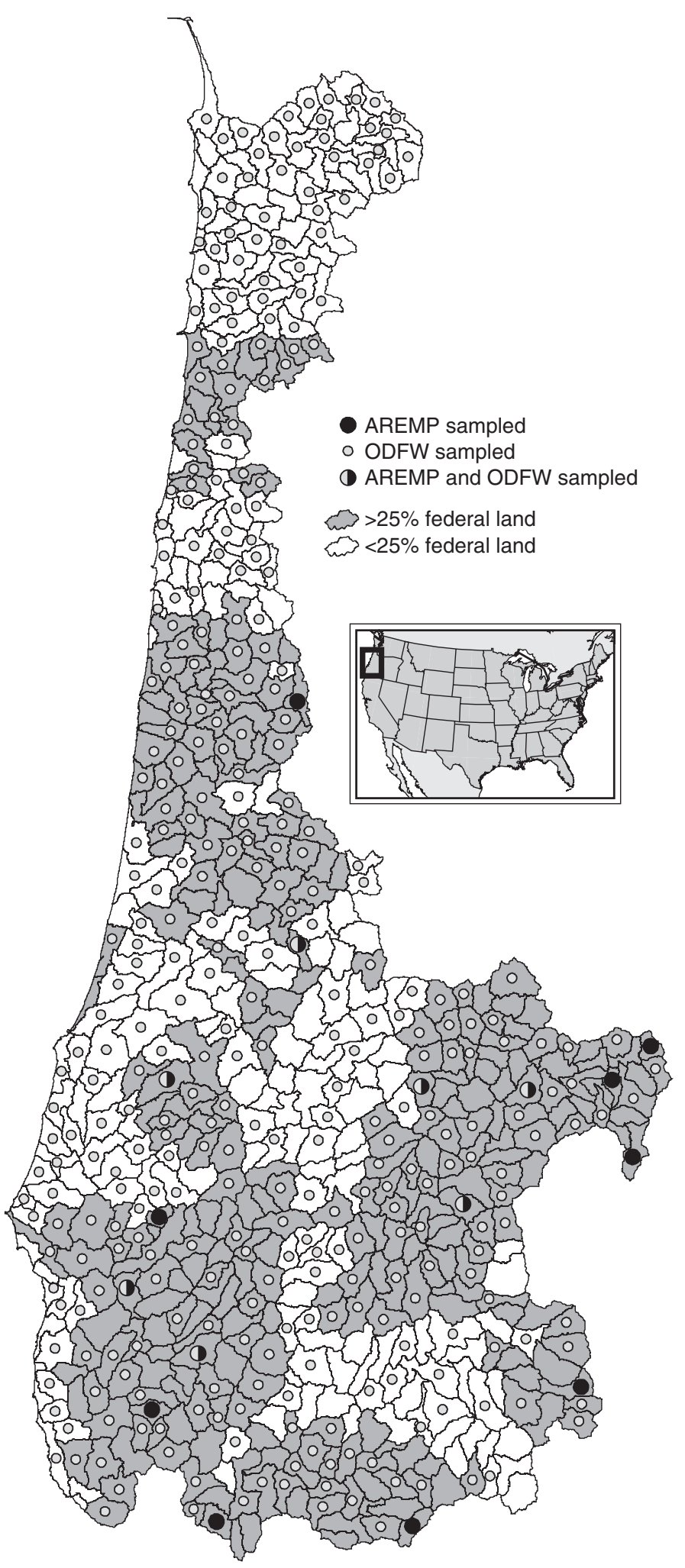

FIGURE 1. A Map Illustrating the Oregon Department of Fish and Wildlife (ODFW) Domain. Shaded area indicates the Aquatic and Riparian Effectiveness Monitoring Program (AREMP) domain within the ODFW domain. Polygons are the hydrologic units (HUs) making up the respective domains with dots indicating which HUs were sampled by which agency (as indicated in the legend). of selecting any location on the network must be determined. For the two stage AREMP design, HUs were first selected, then sites within HUs. Combining the two surveys requires that the two stage cluster structure be preserved. This is accomplished as follows. First, the probability of selecting a stream site within a $\mathrm{HU}$ is the number of sites selected within a HU divided by the stream length within a HU. The number of sites is the combined number of sites from ODFW and AREMP. This is an application of equation 1 assuming post-stratification. AREMP and ODFW sites were both selected with equal probability across stream orders; if this were not the case the process would be similar but involve using weighted stream lengths. Second, the probability of selecting a HU is determined as in the first case. The combined design is a two-stage cluster design where the first stage is an unequal probability sample of HUs and the second stage is an equal probability sample of sites within a HU (although the equal probability differs across HUs). Estimates (cumulative distrubution functions (CDFs), means, medians, etc.) and their uncertainties use this two stage design to account for the fact that sites are clustered, and not spread out over the landscape. It would not be appropriate to determine the probability of selecting a site within the greater than $25 \%$ federal land by dividing the number of AREMP and ODFW sites by the total stream length. This would ignore the cluster aspect of AREMP resulting in incorrect variance estimates.

One physical habitat attribute, the number of pools per $\mathrm{km}$ (pools $/ \mathrm{km})$, is used to illustrate combining data from the two surveys. Both agencies used the same protocol for determining pools $/ \mathrm{km}$ (Moore, et al., 1997; Gallo, 2005). The specific kinds of questions each agency might ask regarding pools $/ \mathrm{km}$ include: what fraction or how many stream kilometers exceed a particular criterion for pools $/ \mathrm{km}$ (ODFW)? How many stream kilometers have pools $/ \mathrm{km}$ lower than a particular value, such as 15 pools $/ \mathrm{km}$ (ODFW)? What is the average pools $/ \mathrm{km}$ in the population of AREMP HUs? In how many HUs does the average pools $/ \mathrm{km}$ exceed 15? The types of questions reflect the two agencies slightly different perspectives on interpretation of the monitoring data. In the case of AREMP, the average pools $/ \mathrm{km}$ could be used as an input to the decision support model that aggregates information across multiple indicators to arrive at an overall HU condition score. ODFW might have an interest in tracking the trends in the proportion of the stream network with $>15$ pools $/ \mathrm{km}$ over time in each monitoring area. The purpose here is strictly to illustrate how the design framework facilitates the process for combining data rather than debating the merits of interpreting data one way or 
another. Thus, the goal is to add to the ODFW database indicator scores taken at AREMP sites (i.e., effectively increase the ODFW sample size by the number of AREMP sites), and add to the AREMP database indicator scores taken at ODFW sites that fall within the AREMP domain.

Cumulative distribution functions (CDFs) are used to summarize data because these summaries contain more information about the regional inference than summary statistics alone (e.g., mean, median, variance, etc.). In addition, summary statistics can be derived by interpreting the graphs, or can be calculated from the data. As illustrated in Figure 2, these CDFs are drawn from an ordered list of the indicator scores with their respective weights (scaled to a total relative weight of 100). Each data point is plotted as follows: the $x$-axis is the indicator score; the $y$-axis is the cumulative weight (as a percent or as scaled to 100). Step heights between points represent the relative weight for a particular site. In this way, the first point ( 1.5 pools $/ \mathrm{km}, 6.23 \mathrm{cum} \%)$ represents the weight associated with that site's score; the second point (8.9 pools $/ \mathrm{km}$; 12.4 cum \%) represents the additional weight (cumulative weight) associated with the second site's score, and so on. Cumulative percent should sum to 100 for the entire resource. Dotted lines are the $95 \%$ confidence bounds representing sampling uncertainty. Stevens and Olsen (2003) and Stevens and Olsen (2004) describe the procedure used to estimate uncertainty and confidence limits for the CDFs. Algorithms for completing the computations are available at the website http://www.epa.gov/

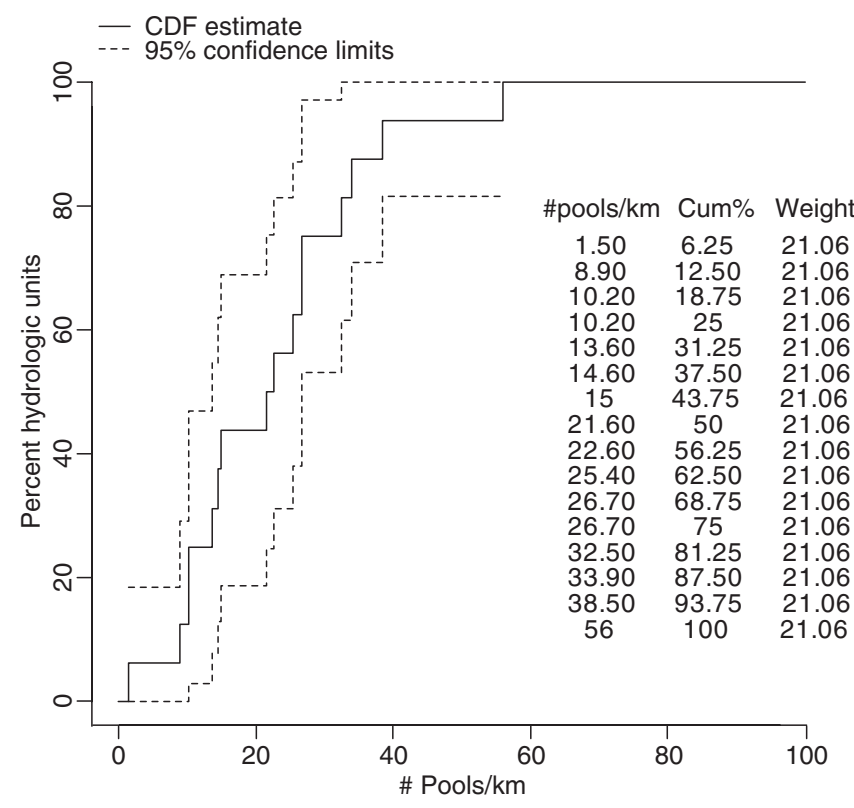

FIGURE 2. An Illustration of the Compilation of Data Into the Cumulative Distribution Functions Used as Data Summaries. nheerl/arm as " $R$ " functions. " $R$ " is a free statistical package ( $R$ Development Core Team, 2004) based on the same "S" statistical language as the commercially available S-Plus. Instructions for downloading "R" are available at the above website. These summaries are presented for each survey separately, then in combination. Specifically, to combine data, the weights (or steps) in the CDF are recalculated to represent combining the sites into the larger sample.

\section{CASE STUDY RESULTS}

\section{Estimating the Stream Pools $/ \mathrm{km}$}

The frequency distribution of pools $/ \mathrm{km}$ in stream channels can be summarized as the proportion of the stream network with pools $/ \mathrm{km}$ higher or lower than particular values. These summaries can be organized in a variety of ways. In the following "AREMP" and "ODFW" refer to the pools $/ \mathrm{km}$ data collected by the respective agencies. The following illustrates individual survey results and their combination (Figure 3; Table 1): AREMP sites in the AREMP domain (Figure 3a); ODFW sites in AREMP domain (Figure 3b); AREMP sites+ODFW sites in AREMP domain (Figure 3c); ODFW sites in the ODFW domain excluding the AREMP domain (Figure 3d). The combination of AREMP and ODFW sites across the entire ODFW domain is not shown because differences between that graph and ODFW alone are visually indistinguishable. The number of AREMP sites added to the sample is small in relation to the ODFW sample size and causes only slight changes to the CDF. Uncertainty bounds demonstrate the higher precision achieved when the large ODFW sample is added to the relatively modest AREMP sample (i.e., compare Figure 3c with Figure 3a), illustrating the benefits of interagency data sharing, especially to AREMP. To aid the comparisons, each graph illustrates the median pools $/ \mathrm{km}$ and its $95 \%$ confidence interval; see also Table 1. Figure 3c and 3d compare the distribution of pools $/ \mathrm{km}$ in streams in the AREMP domain with the distribution of pools $/ \mathrm{km}$ in streams outside the AREMP domain. In the case of pools $/ \mathrm{km}$, there is no apparent difference in the median pools $/ \mathrm{km}$ between these two domains.

\section{Estimating the Average Pools/km in HUs}

The same data can be organized instead as the frequency distributions of average pools $/ \mathrm{km}$ in HUs; the summary metric for each $\mathrm{HU}$ is the average pools $/ \mathrm{km}$ 
(a)

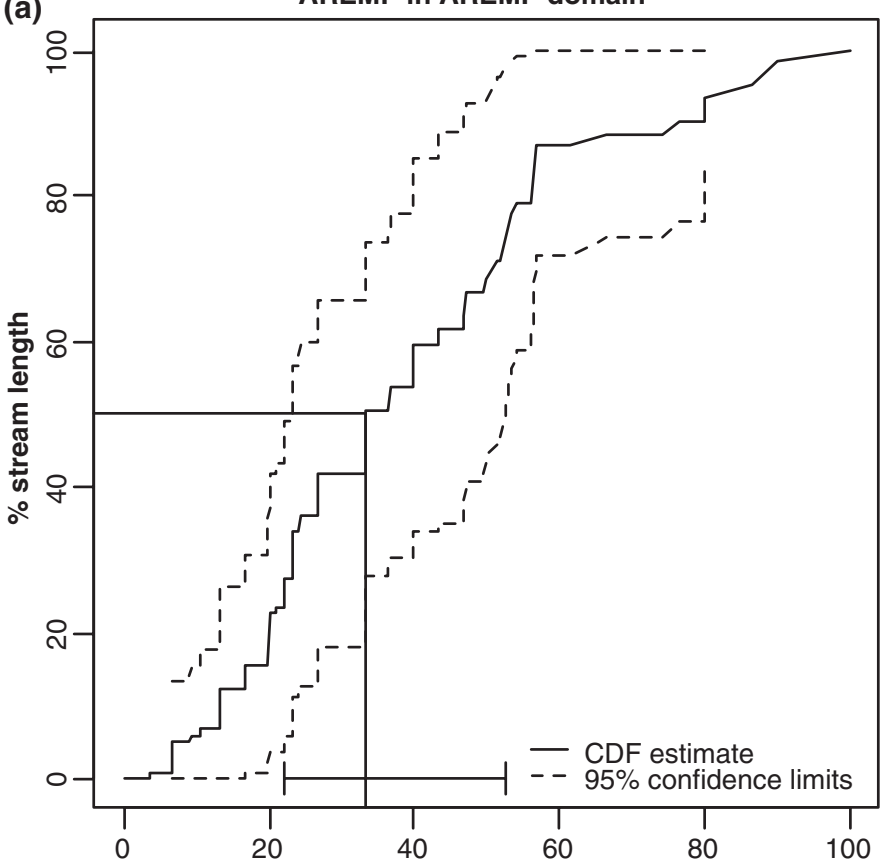

(c)

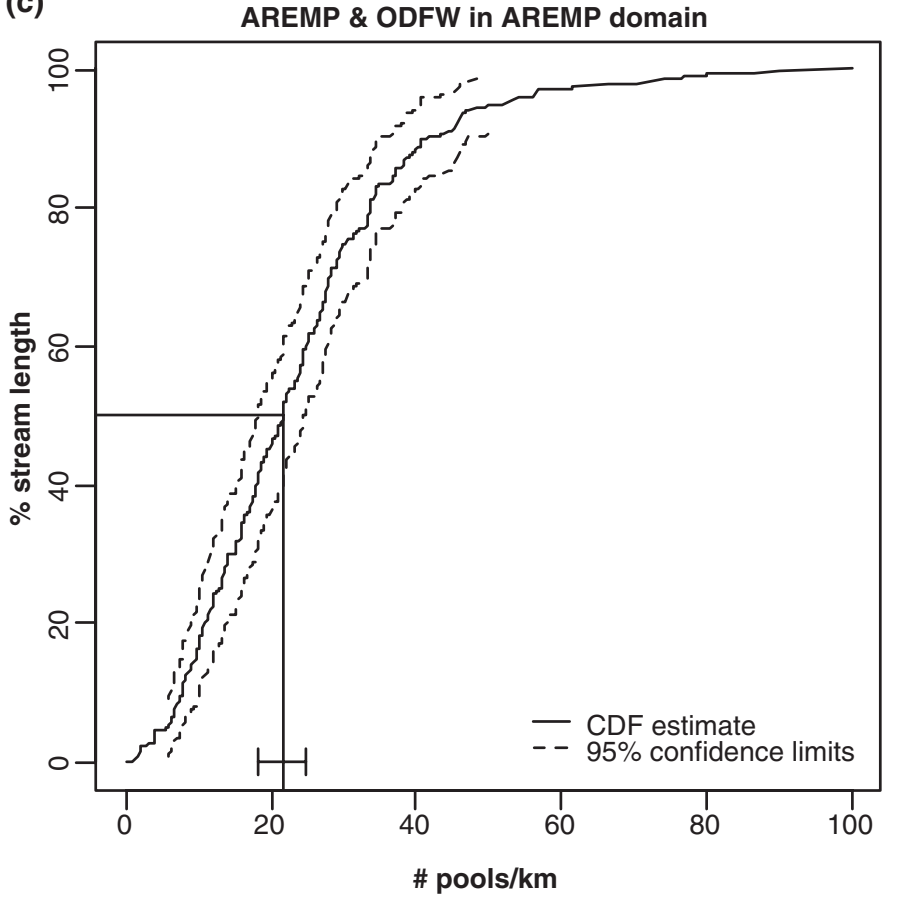

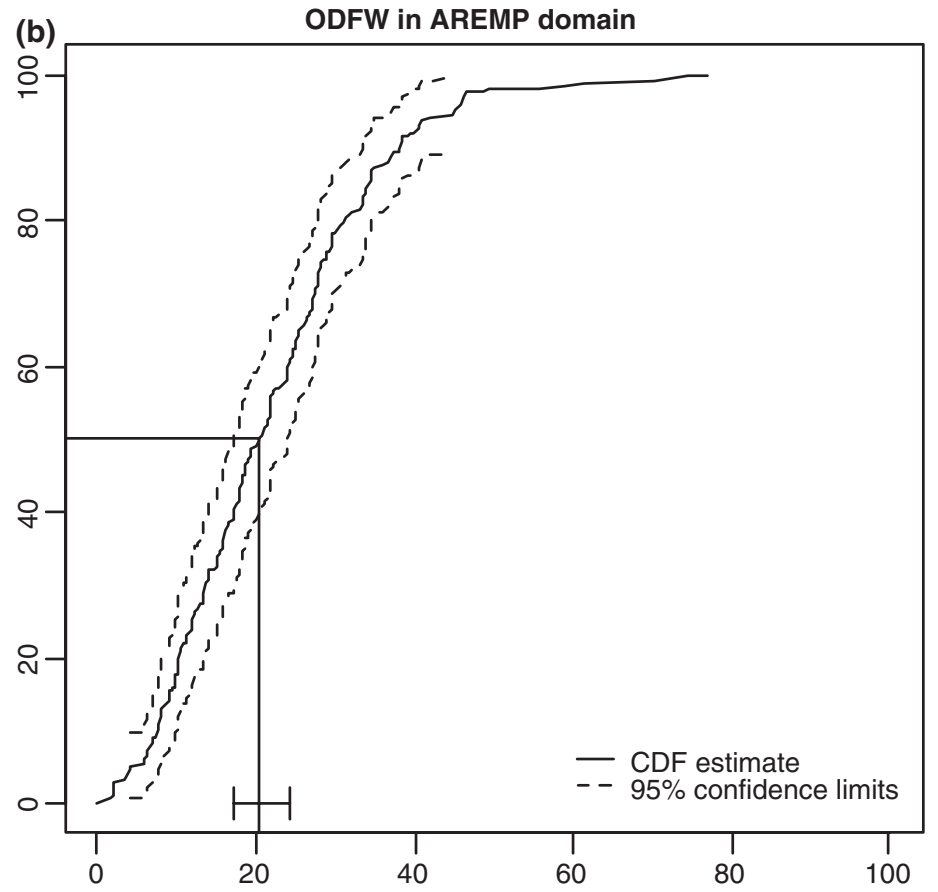

(d)
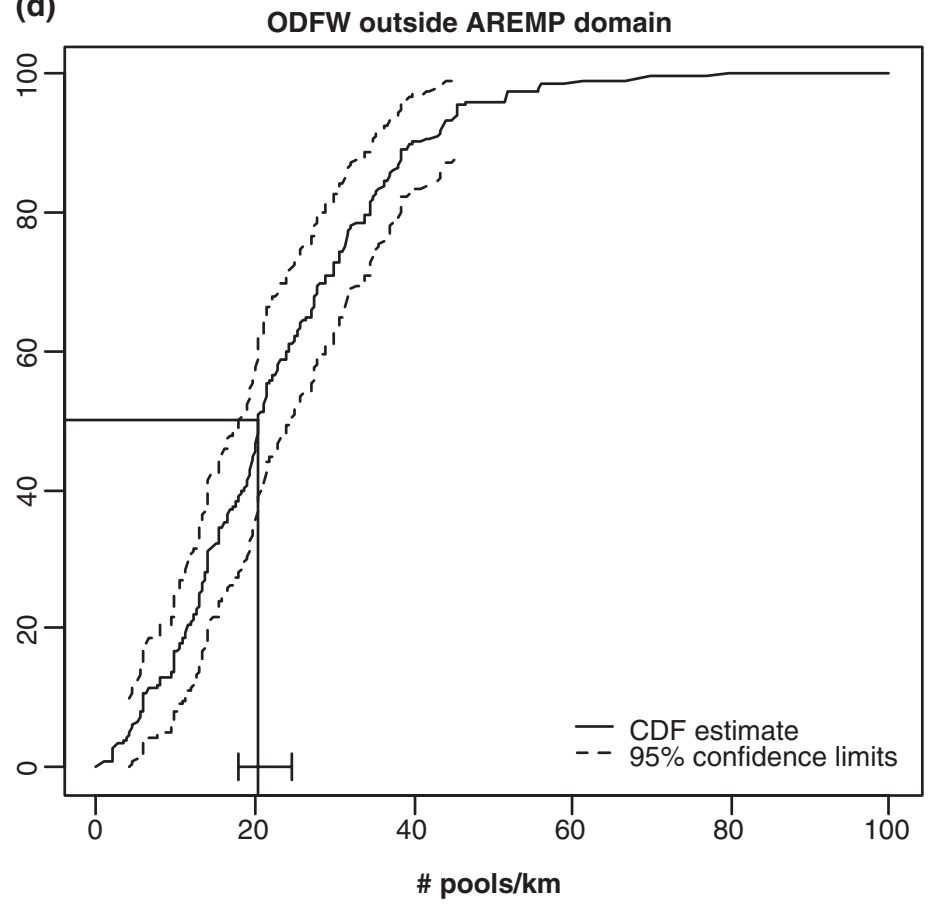

FIGURE 3. Cumulative Distribution Functions Illustrate Combining the Data in Different Ways to Characterize Stream Networks. To aid in comparisons among figures, the median pools $/ \mathrm{km}$ (with $95 \%$ confidence bounds) is indicated with the horizontal and vertical lines.

across the set of stream sites monitored in each HU. The data are summarized with respect to the population of HUs, rather than with respect to the stream network. These CDF summaries are presented in the same combination of ways as for the stream network (Figure 4; Table 1): AREMP in AREMP domain (Figure 4a); ODFW in AREMP domain (Figure 4b); AREMP+ODFW in AREMP domain (Figure 4c); ODFW outside AREMP domain (Figure $4 \mathrm{~d}$ ). The combination of AREMP and ODFW in ODFW domain is not shown for the same reasons stated above. Evaluation of the bounds demonstrates the higher precision obtained if data from both surveys are combined; compare Figure $4 \mathrm{a}$ with Figure 4c. Medians, with 95\% confidence bounds, are illustrated as well, and summarized in Table 1). 
TABLE 1. Medians and 95\% Confidence Bounds Corresponding to Figures 3a-d and 4a-d.

\begin{tabular}{lccc}
\hline Figure & Median & LCL & UCL \\
\hline 3a & 33.3 & 22.2 & 52.5 \\
3b & 20.5 & 17.3 & 24.1 \\
3c & 21.7 & 18.2 & 24.9 \\
3d & 20.5 & 17.8 & 24.6 \\
$4 \mathrm{a}$ & 23.0 & 11.9 & 34.8 \\
$4 \mathrm{~b}$ & 18.0 & 16.8 & 19.9 \\
$4 \mathrm{c}$ & 18.4 & 17.2 & 21.4 \\
$4 \mathrm{~d}$ & 18.5 & 16.6 & 22.0 \\
\hline
\end{tabular}

Clearly, in both cases (stream network or HU perspectives) precision improves dramatically if AREMP incorporates ODFW data into its data summaries regardless of whether it chooses to summarize by $\mathrm{HU}$, or by stream network.

\section{DISCUSSION}

Agencies commonly express a desire to combine data from multiple monitoring programs to make regional estimates of stream network's or watershed's condition, even though the agencies may use and interpret the data in different ways. Although it might be desirable for a single agency to oversee a region-wide monitoring program for aquatic resources analogous to the way agricultural resources are monitored through the National Agricultural Statistical Surveys (Cotter and Nealon, 1987), or the condition of forests is monitored through the Forest Health Monitoring/Forest Inventory and Analysis surveys (Birdsey and Schreuder, 1992; FHM, 1994), such a centralized program does not seem realistic for aquatic resources. Instead, many agencies responsible for overlapping geographic domains of responsibility develop agency specific monitoring programs, while sometimes expressing interest in coordinating their monitoring programs, or at least in combining data from their overlapping programs. Such a combination of data is feasible and can be statistically sound as long as certain aspects of the theory and practice of survey sampling are recognized and adopted.

In many cases, a key difficulty with combining data from different programs is that the monitoring designs have not followed survey sampling principles. For example, sites are often selected judgmentally, or by convenience, with the result that it is difficult if not impossible to determine the selection bias. At the same time, sample weights (or inclusion probabilities) cannot be determined easily or defensibly. For these reasons, combining data can yield biased pictures of regional condition. If the principles of survey sampling are incorporated into the monitoring design, the possibility of combining results for regional estimates is substantially improved.

Adopting shared stream sample survey techniques implies achieving agreement on three important aspects of survey design including: selection and use of consistent frame materials; identification of common sample units or elements; and the use of probability methods for site selection from frame.

\section{Frame Materials}

Although agency-specific target populations might differ with respect to geographic domain or stream type, it is feasible to consider using agreed-upon consistent frame materials to represent the potential target populations. One agency might only focus on those streams in a specific river basin that are populated by a particular species; other agencies might be interested in the water quality of all streams in a variety of river basins; still others might be interested in streams under a specific agency's control, such as a national forest or tribal lands. If there is a shared interest in evaluating stream network condition, either alone or as part of evaluating watershed condition, a consistent representation of the stream network and the watersheds covering the broadest domain is necessary to be able to integrate the monitoring data. The frame material should cover the domains of interest, should represent the "on the ground" resource to be monitored, and be relatively error free. For stream networks, commonly available frame materials for the coterminous United States can be derived from the digital versions of the USGS 1:100,000 scale hydrographic maps available as the National Hydrography Database (http://eros.usgs.gov/ geodata).

This database contains a reasonably useful representation of most stream networks, but it contains errors that should be recognized. Stream density sometimes varies from map to map. For some agency interests, the frame might be incomplete; parts of the stream network might not be represented, or watersheds of interest might not be included. The digital coverage might be inaccurate (e.g., streams mapped as perennial might be dry most of the time, or the cartographer might have erred in mapping stream channels where none exist, or streams indicated as perennial might have been submerged by dam construction since the map was created). Although these errors occur, this database is what is available at broad regional scales. In some areas, effort is underway to develop more consistent, detailed stream networks (for example, based on 1:24,000 scale 
(a)

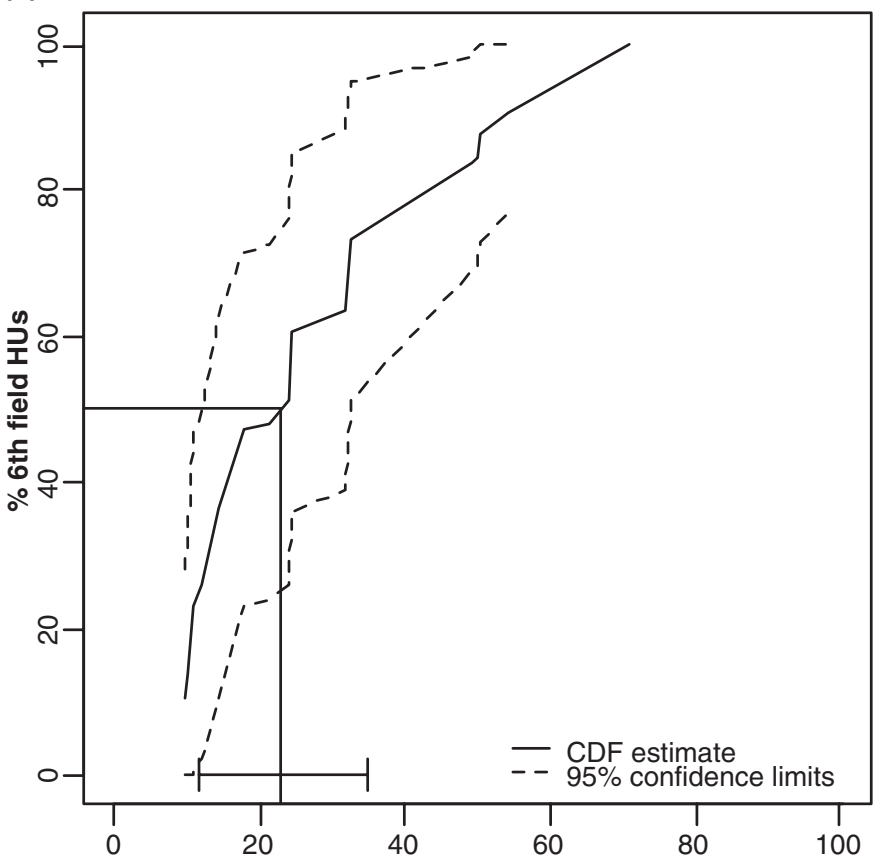

(c)

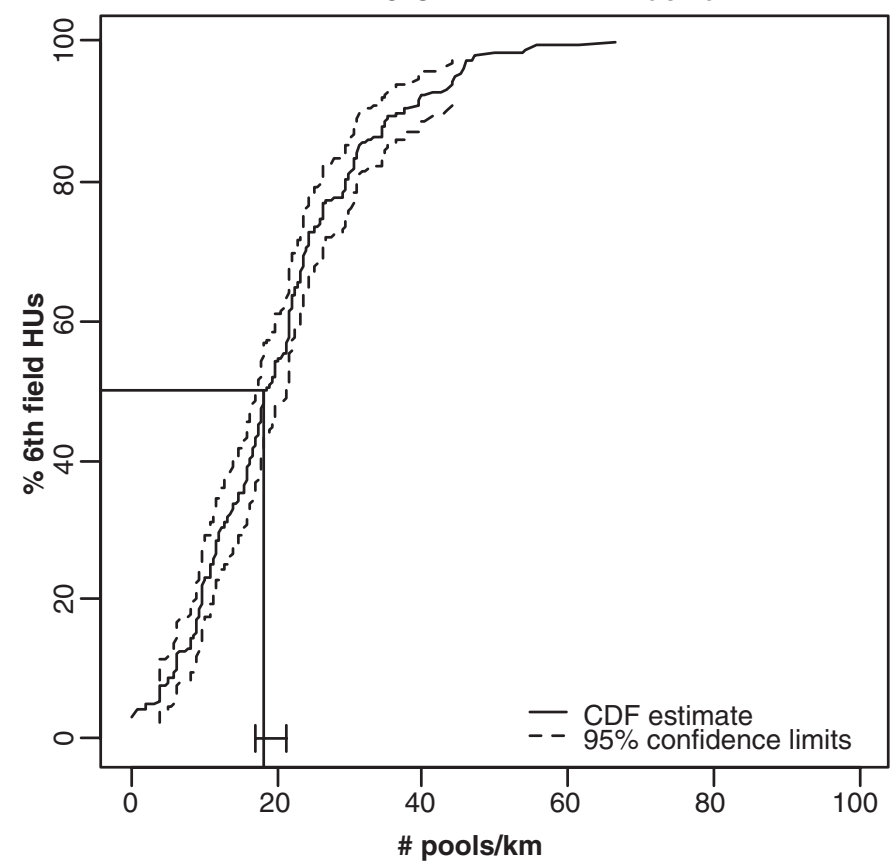

(b) ODFW in AREMP domain

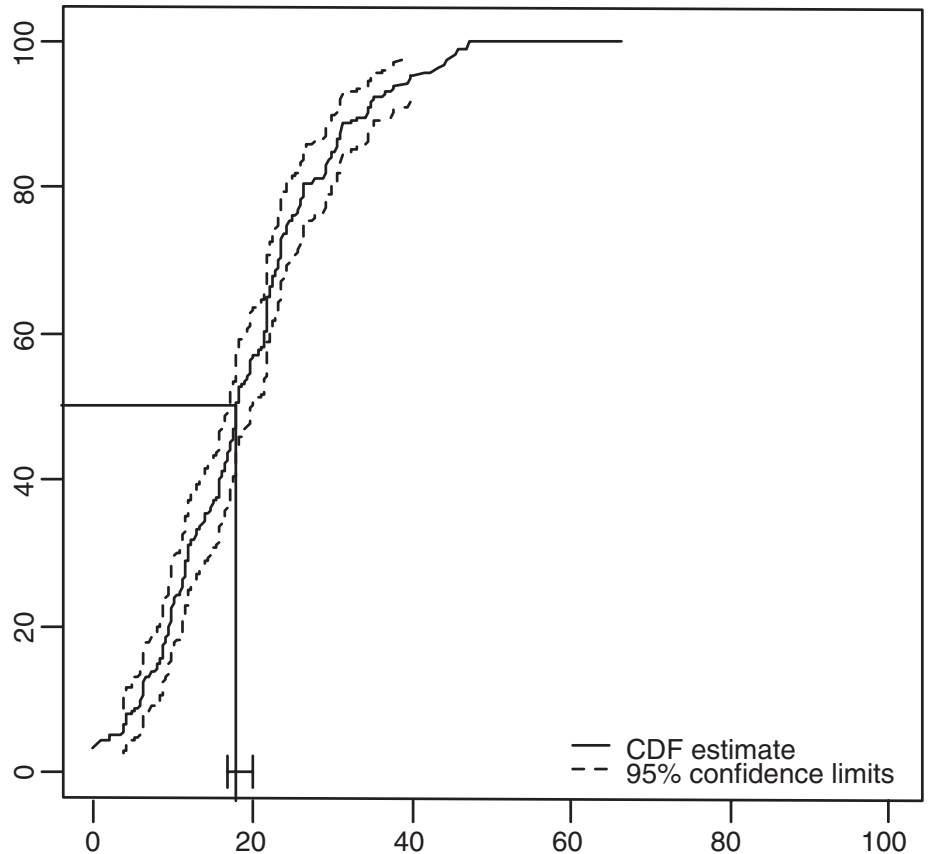

(d)

ODFW outside AREMP domain

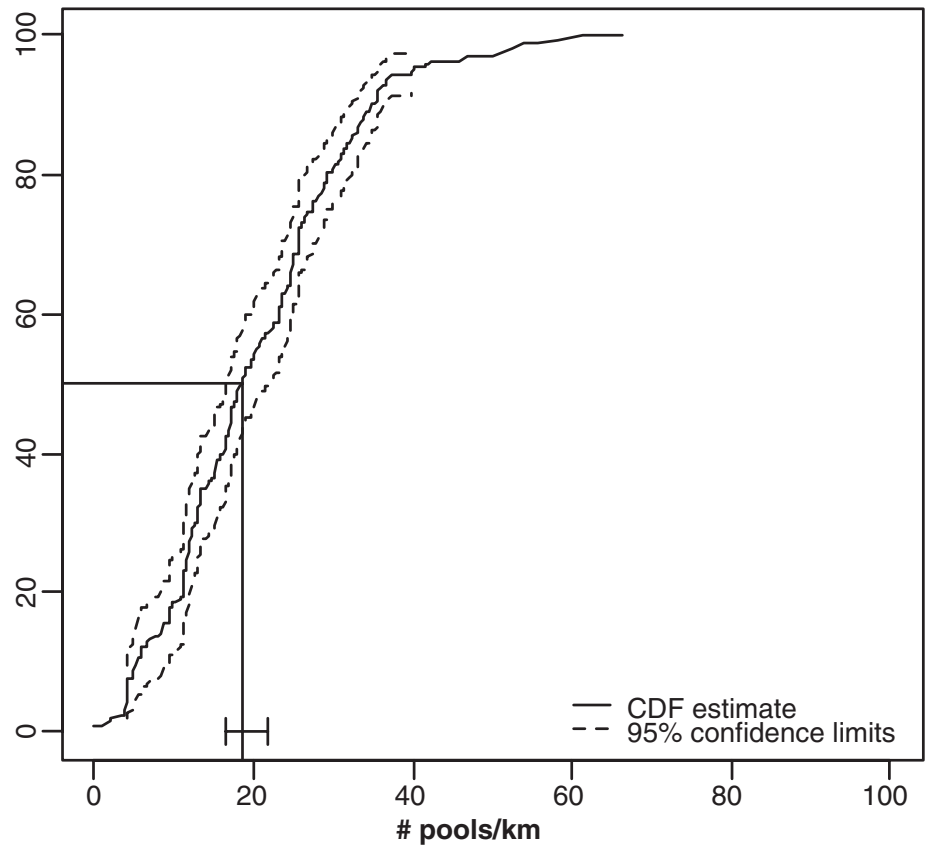

FIGURE 4. Cumulative Distribution Functions Illustrate Combining the Data in Different Ways to Characterize the Average Pools/km in Hydrologic Units. To aid in comparisons among figures, the median pools $/ \mathrm{km}$ (with $95 \%$ confidence bounds) is indicated with the horizontal and vertical lines.

hydrography, or based on digital elevation models), and these can be used if an entire region of interest is covered. Managing the frame errors can be incorporated as part of the survey design. Errors of omission can be accommodated as part of the ongoing survey by updating the frame as unmapped target channels are identified and digitized.
With respect to frame materials for watersheds, the USGS hydrographic accounting system and the resultant maps (Seaber et al., 1987) have been used to define watersheds as sample units (i.e., down to fourth field, or eight-digit, HUs), with some important caveats discussed later. However, in many cases, agencies are interested in smaller watersheds. There- 
fore a hierarchical decomposition of the landscape into finer and finer hydrographic units is required. Guidelines are available for this process (Legleiter, 2001), but relatively small parts of the country have been mapped to these finer scales in a consistent manner. Nonetheless, for areas of interest, these units could be delineated on an as needed basis, following the guidelines.

\section{Population Elements}

Three kinds of population elements make up a target population of stream networks or watersheds: (1) points on the stream network; (2) stream segments or reaches; and (3) watersheds. Using points on stream networks is based on the perspective that the target stream network is an extensive resource consisting of an infinite number of points, each of which has the possibility of being selected. Adopting this version of a sample element also requires specifying a sampling support area (Cressie, 1991; Stevens and Urquhart, 1999): most indicators cannot be measured at a specific point on a stream but rather require making measurements in the vicinity of the point. The measurements made at the point (e.g., temperature), or in the vicinity of the point (e.g., fish species richness), are used as an estimate of the attribute associated with that point. Conceptually, the attribute is assumed to change continuously over the stream network. As the support area for the measurement increases, the measured process is smoothed. This concept is not unusual in the natural resource sampling literature in that both NASS (Cotter and Nealon, 1987) and FIA/FHM (Birdsey and Schreuder, 1992; FHM, 1994) use a point sample with a support area around the point where the measurements are taken. It is not uncommon for stream sampling protocols to specify a support area even if survey sampling techniques are not adopted. For example, the following have been used: a fixed distance such as 500 or $1000 \mathrm{~m}$ (Jones and Moore, 1999; Moore et al., 1997); or a distance expressed in terms of channel width (Lazorchak et al., 1998; Roper et al., 2003). Certainly, specifying the reach length is an important part of the monitoring design.

The use of discrete stream reaches as population elements involves defining the rules to be used for decomposing the continuous network into discrete pieces. For example, stream reaches have been defined as the continuous stream length between confluences, or between well-defined geomorphic features, or a combination of both. This process converts a continuous population into a discrete population of well-defined population elements. In this case, the stream network has discrete changes in attribute values from one segment to the next. Measurements in each segment are used as an estimate associated with that segment. The method used to decompose the continuous resource should be applied to the entire resource so that the target population can be defined as the collection of discrete population elements.

Using watersheds as population elements poses some interesting challenges. A basic interpretation of a watershed is a well-defined unit delineated by drainage divides and network outlets such as at confluences of stream channels. However, drainage boundaries can be delineated for any point on a stream network. Watersheds can be thought of as a continuous resource in the same manner that the stream channel network can be considered a continuous resource. For various practical purposes, watershed units of a particular size range are specified. For example, first-order watersheds in a specific geographic domain might be surveyed, as Ator et al. (2003) have done. Or the USGS hydrologic accounting unit framework can be used to specify population elements. When using watersheds as population elements, it is essential to define the level in this hierarchy that would be used, i.e., the collection of fourth or sixth field HUs. The sample then consists of a probability selection of a specified number of HUs, so inferences can be made to the entire collection of HUs of the specified size.

One underappreciated aspect of the use of HUs is that the landscape does not decompose into a population of complete watersheds. Roughly $50 \%$ of any area consists of complete, or discrete, watersheds of the selected size range, that is, accounting units that originate with headwaters and drain through a single outlet (Omernik, 2003; Detenbeck et al., 2004). The rest of the landscape contains areas of similar size but with a mix of direct drainages to large channels, to coastal areas, to closed basins, or to subsurface flow; often these "composite" watersheds contain an inflow from upstream. An instructive exercise is to examine a map of these HUs along with the stream traces to evaluate the units that meet the traditional definition of a watershed (with headwater channels joining to form a single outlet) and these other units that usually have both a main channel inlet and outlet with additional small channels or might be several small watersheds draining into a coastal area. Careful consideration is needed if the hydrologic accounting system is to be used as a basis for defining a population of watersheds.

\section{Randomization in Site Selection Process}

The rule that all population elements have a chance of being selected in the sample is as relevant for aquatic resource surveys as it is for any other resource. This does not mean that each element must have the 
same likelihood of being selected, rather that there is a known, positive chance that each be selected. Simple random sampling is one process of sample selection; stratification and other methods that result in variable selection probabilities can also be used. However, it is critical that the selection probabilities be known because they are used in making inferences from the sample to the population as a whole through the frequency distributions and sampling uncertainty. Stevens and Olsen $(1999,2004)$ describe a sample selection approach widely applicable to various types of natural resources, e.g., linear resources like stream networks; resources defined as discrete units like lakes or watersheds; continuous two dimensional resources like estuaries. This approach allows great flexibility in the design choices yet maintains the fundamental principles of survey sampling. In addition, the process takes advantage of the spatial structure (if present) of the surveyed attributes which results in greater precision than that obtained with a simple (or stratified) random sample (Stevens and Olsen, 2004). One of the important outcomes of meeting the criterion that each element has known probability of selection or inclusion in the sample is that the results from different surveys can be combined by recalculating the sample weights, given a consistent frame and definition of population elements.

\section{Concluding Comments}

The survey design framework described along with adherence to basic survey design principles allows interested agencies the opportunity to integrate their monitoring data in a statistically sound manner. Furthermore, sharing data yields cost savings, promotes communication, and integrates results. However, adopting a survey design framework also implies a commitment to building the necessary infrastructure to support integrated monitoring. At a minimum, this includes specifying common and disparate objectives clearly, creating the common frame materials, establishing database management systems to facilitate data sharing, and developing and using common protocols for common indicators. Sharing data has been difficult, perhaps in part because no unifying design framework has been in place to facilitate the needed level of communication and integration. Adoption of the principles outlined here would stimulate such integration.

\section{ACKNOWLEDGMENTS}

The following people are acknowledged for their assistance: Leska Fore and Dave Marmorek for insightful reviews of the manu- script; Sue Pierson for graphics assistance; numerous field crew members whose field data collection made this possible; and three anonymous reviewers. David Larsen was employed by the U.S. EPA and Thomas Kincaid by Dynamac International Corp. at the time of preparation of the manuscript. The information in this document has been funded in part by the U.S. Environmental Protection Agency. It has been subjected to review by the National Health and Environmental Effects Research Laboratory and approved for publication. Approval does not signify that the contents reflect the views of the Agency, nor does mention of trade names or commercial products constitute endorsement or recommendation for use.

\section{REFERENCES}

Ator, S.W., A.R. Olsen, A.M. Pitchford, and J.M. Denver, 2003. Application of a Multipurpose Unequal Probability Stream Survey in the Mid-Atlantic Coastal Plain. Journal of the American Water Resources Association 39(4):872-885.

Birdsey, R.A. and H.T. Schreuder, 1992. An Overview of Forest Inventory and Analysis Estimation Procedures in the Eastern United States-With an Emphasis on Components of Change. Gen. Tech. Rept. RM-214, U.S. Department of Agriculture, Forest Service, Rocky Mountain Forest and Range Experiment Station, Ft. Collins, Colorado (FIA point sample).

Cochran, W.G., 1987. Sampling Techniques, 3rd edn. John Wiley \& Sons, New York, 428 pp.

Converse, J.M., 1987. Survey Research in the United States: Roots and Emergence 1890-1960. University of California Press, Berkeley, California. 564 pp.

Cotter, J. and J. Nealon, 1987. Area Frame Design for Agricultural Surveys. U.S. Department of Agriculture, National Agricultural Statistics Service, Washington, District of Columbia.

Cressie, N., 1991. Statistics for Spatial Data. John Wiley \& Sons. New York.

Detenbeck, N.E., D. Cincotta, J.M. Denver, S.K. Greenlee, A.R. Olsen, and A.M. Pitchford, 2005. Watershed-based Survey Designs. Environmental Monitoring and Assessment 103:59-81.

Forest Health Monitoring Program (FHM). 1994. Forest Health Monitoring: a National Strategic Plan. U.S. Department of Agriculture, Forest Service, Research Triangle Park, North Carolina, $254 \mathrm{pp}$.

Gallo, K., S.H. Lanigan, P. Eldred, S.N. Gordon, and C. Moyer, 2005. Northwest Forest Plan-The First 10 Years (1994-2003): Preliminary Assessment of the Condition of Watersheds. Gen. Tech. Rep. PNW-GTR-647. U.S. Department of Agriculture, Forest Service, Pacific Northwest Research Station, Portland, Oregon. $133 \mathrm{pp}$.

Herlihy, A.T., D.P. Larsen, S.G. Paulsen, N.S. Urquhart, and B.J. Rosenbaum, 2000. Designing a Spatially Balanced Randomized Site Selection Process for Regional Stream Surveys -The EMAP Mid-Atlantic Pilot Study. Environmental Monitoring and Assessment 63:95-113.

Johnson, D.H., N. Pittman, E. Wilder, J.A. Silver, R.W. Plotnikoff, B.C. Mason, K.K. Jones, P. Roger, T.A. O'Neil, and C. Barrett, 2001. Inventory and Monitoring of Salmon Habitat in the Pacific Northwest - Directory and Synthesis of Protocols for Management/Research and Volunteers in Washington, Oregon, Idaho, Montana, and British Columbia. Washington Department of Fish and Wildlife, Olympia, Washington, $212 \mathrm{pp}$.

Jones, K.K. and K.M.S. Moore, 1999. Habitat Assessment in Coastal Basins in Oregon: Implications for Coho Salmon Production and Habitat Restoration. In: Sustainable Fisheries Management, E.E. Knudsen, C.R. Steward, D.D. McDonald, J.E. Williams, and D.W. Riser (Editors). CRC Press, New York, pp. 329-340. 
Kish, L., 1965. Survey Sampling. Wiley, New York.

Lazorchak J.M., D.J. Klemm, and D.V. Peck (Editors), 1998. Environmental Monitoring and Assessment Program - Surface Waters: Field Operations and Methods for Measuring the Ecological Condition of Wadeable Streams. EPA/620/R-94/004F. U.S. Environmental Protection Agency, Office of Research and Development, Washington, District of Columbia. 211 pp. plus appendices.

Legleiter, K.J., 2001. Interagency Development of National Watershed and Subwatershed Hydrologic Units. Paper 492. In: Proceedings, 2001 ESRI International User's Conference, July 9-13, 2001. San Diego, California.

Lohr, S.L., 1999. Sampling: Design and Analysis. Duxbury Press, Pacific Grove, California.

Montgomery, D.R., G.E. Grant, and K. Sullivan, 1995. Watershed Analysis as a Framework for Implementing Ecosystem Management. Water Resources Bulletin 31:369-386.

Moore, K.M.S., K.K. Jones and J.M. Dambacher, 1997. Methods for Stream Habitat Surveys. Oregon Department of Fish and Wildlife, Information Report 97-4, Salem, Oregon, 40 pp.

Nicholas, J.W., 1997. The Oregon Plan for Salmon and Watersheds: Oregon Coastal Salmon Restoration Initiative. State of Oregon, Salem, Oregon.

Olsen, A.R., J. Sedransk, E. Edwards, C.A. Gotway, and W. Liggett, 1999. Statistical Issues for Monitoring Ecological and Natural Resources in the United States. Environmental Monitoring and Assessment 54:1-45.

Omernik, J.M., 2003. The Misuse of the Hydrologic Unit Maps for Extrapolation, Reporting and Ecosystem Management. Journal of the American Water Resources Association 39:563-573.

Oregon Plan, 1997. The Oregon Plan for Salmon and Watersheds (Consisting of the Oregon Coastal Salmon Restoration Initiative, March 10, 1997 and as Amended with the Steelhead Supplement, December 1997). Governor's Natural Resources Office, State of Oregon, Salem, Oregon.

Overton, J.M., T.C. Young, and W.S. Overton, 1993. Using 'Found' Data to Augment a Probability Sample: Procedure and a Case Study. Environmental Monitoring and Assessment 26:65-83.

$\mathrm{R}$ Development Core Team. 2004. R: A language and environment for statistical computing. R Foundation for Statistical Computing, Vienna, Austria. ISBN 3-900051-07-0. http://www.Rproject.org.

Reeves, G.H., D.B. Hohler, D.P. Larsen, D.E. Busch, K. Krratz, K. Reynolds, K.F. Stein, T. Atzet, P. Hays, and M. Tehan, 2004. Effectiveness Monitoring for the Aquatic and Riparian Component of the Northwest Forest Plan: Conceptual Framework and Options. Gen. Tech. Rep. PNW-GTR-577. U.S. Department of Agriculture, Forest Service, Pacific Northwest Research Station. Portland, Oregon. $71 \mathrm{pp}$.

Roper, B.B., J.L. Kershner, and R.C. Henderson, 2003. The Value of Using Permanent Sites when Evaluating Stream Attributes at the Reach Scale. Journal of Freshwater Ecology 18:585-592.

Seaber, P.R., P. Kapinos, and G.L. Knapp, 1987. Hydrologic Unit Maps. U.S. Geological Survey Water-Supply Paper 2294. U.S. Govt. Printing Office, Washington, DC. 63 pp.

Stevens, D.L., Jr., 1997. Variable Density Grid-based Sampling Designs for Continuous Spatial Populations. Environmetrics 8:167-195.

Stevens, D.L., Jr., 2002. Sampling Design and Statistical Analysis Methods for the Integrated Biological and Physical Monitoring of Oregon Streams. Report Number: OPSW-ODFW-2002-07. Oregon Department of Fish and Wildlife, Portland, Oregon.

Stevens, D.L., Jr. and A.R. Olsen, 1999. Spatially Restricted Surveys over Time for Aquatic Resources. Journal of Agricultural, Biological, and Environmental Statistics 4:415-428.

Stevens, D.L., Jr. and A.R. Olsen, 2003. Variance Estimation for Spatially Balanced Samples of Environmental Resources. Environmetrics 14:593-610.
Stevens, D.L., Jr. and A.R. Olsen, 2004. Spatially Balanced Sampling of Natural Resources. Journal of the American Statistical Association 99:262-278.

Stevens, D.L., Jr. and Urquhart N.S., 1999. Response Designs and Support Regions in Sampling Continuous Domains. Environmetrics 11:13-41.

Thompson, S.K., 1992. Sampling. John Wiley \& Sons, New York. $360 \mathrm{pp}$. 